\title{
Experiencia de una unidad aerodigestiva pediátrica en Latinoamérica
}

\section{Case management experience in a pediatric aerodigestive unit in Latin-America}

\author{
Rosina López ${ }^{1 *}$, Maynor Bravo², Shirley Cuan², Edgar Beltetón ${ }^{3}$, Christian Farrington ${ }^{3}$
}

\section{Resumen}

Introducción: las unidades aerodigestivas proveen un cuidado complejo coordinado a pacientes pediátricos con condiciones congénitas o adquiridas de grados variables que afectan la vía respiratoria, el tracto gastrointestinal, la deglución y el crecimiento. La primera unidad aerodigestiva de Guatemala fue fundada en el año 2018 en un hospital privado, y por ser la única de su tipo en el país, no se tenían datos locales al respecto. Pacientes y métodos: se llevó a cabo un estudio retrospectivo cuantitativo; se analizó el total de casos reportados en la unidad aerodigestiva pediátrica desde el 1 de enero de 2018 hasta el 31 de mayo de 2019, se contó con un total de 69 casos. Resultados: el 79\% de los pacientes presentaron más de una patología de diferente etiología. La indicación principal para realizar la triple endoscopia fue tos crónica. Los diagnósticos encontrados como comorbilidades con mayor frecuencia fueron bronquitis bacteriana recurrente y enfermedad por reflujo gastroesofágico. Conclusión: la triple endoscopia es útil tanto en el diagnóstico como en la toma de decisiones en el manejo de las patologías aerodigestivas pediátricas de alta complejidad. Es una herramienta útil que pone en evidencia que múltiples etiologías pueden contribuir a síntomas crónicos de la vía aérea, y que estas pueden pasarse por alto si se realiza un solo procedimiento.

Palabras clave: unidad aerodigestiva; broncoscopia pediátrica; endoscopia; laringoscopia; pediatría; ciudad de Guatemala.

\begin{abstract}
Introduction: Aerodigestive programs provide coordinated interdisciplinary care to pediatric patients with complex congenital or acquired conditions affecting breathing, the gastrointestinal tract, swallowing, and growth. The first aerodigestive program in Guatemala was founded in the year 2018 in a private hospital and, since it's one of its kind in the country, there was no local data reported. Patients and methods: A total of 69 cases of children who were evaluated at the aerodigestive program from January $1^{\text {st }}, 2018$ to May $31^{\text {st }}, 2019$, were retrospectively analyzed in a quantitative study. Results: $79 \%$ of patients presented more than one pathology from different etiology. Overall the main indication for the triple scope was chronic cough. The findings reported that recurrent bacterial bronchitis and gastroesophageal reflux were the most common comorbidities present in the same patient. Conclusion: The triple scope procedure is a useful investigative tool for patients with recalcitrant aero-digestive complaints. In particular, the triple scope can yield more than one specialty-specific diagnosis normally missed by one procedure.
\end{abstract}

Keywords: aerodigestive unit; pediatric bronchoscopy; endoscopy; laryngoscopy; pediatrics; Guatemala City.

Fecha de Envío: 27 de marzo de 2020 - Fecha de aceptación: 21 de agosto de 2020

\section{Introducción}

Los avances en el cuidado de los pacientes pediátricos han incrementado la población de niños con condiciones crónicas multiorgánicas complejas. El cuidado de estos pacientes es costoso y se acompaña de múltiples procedimientos, un gran soporte en la tecnología con un equipo multidisciplinario, así como hospitalizaciones frecuentes. Este debe ser consistente, efectivo, costo eficiente $y$, sobre todo, centrado en el paciente. Por esto, es necesario un equipo coordinado e integrado que provea un adecuado manejo a esta población (Collaco et al., 2015; Fracchia et al., 2016; Boesch et al., 2018).

(1) Facultad de Medicina. Universidad Francisco Marroquín. Ciudad de Guatemala.

(2) Departamento de Pediatría Hospital Herrera Llerandi. Ciudad de Guatemala.

(3) Centro Pediátrico de Guatemala.

*Autor de correspondencia: mrlopez@ufm.edu 
Las unidades aerodigestivas proveen un cuidado complejo coordinado a pacientes pediátricos con condiciones congénitas o adquiridas que afectan en grados variables la vía respiratoria, el tracto gastrointestinal, la deglución y el crecimiento (Boesch et al., 2018). Estas condiciones incluyen patologías estructurales o fisiológicas de la vía aérea, enfermedad pulmonar parenquimatosa crónica, daño pulmonar por aspiración o infección, reflujo gastroesofágico, esofagitis eosinofílica, trastornos en la motilidad esofágica, disfagia y problemas con la alimentación. Ejemplos de los desórdenes evaluados en los programas aerodigestivos incluyen: tos crónica, anomalías craneofaciales, estenosis laringotraqueal, infecciones recurrentes, crup, y estridor recurrente, entre otros (Collaco et al., 2015).

La fundación de esta modalidad de clínicas multidisciplinarias tuvo lugar en Cincinnati, Estados Unidos, en el año 1999 y desde entonces existen más de 50 programas aerodigestivos en dicho país (Boesch et al., 2018). Sin embargo, hasta el momento no existe un consenso que estandarice la estructura de estas unidades. No obstante, diversos estudios han demostrado su utilidad con el uso de la triple endoscopia (laringoscopia, broncoscopia y endoscopia digestiva alta: esófago, estómago y primera y segunda porción del duodeno) para llegar a un adecuado diagnóstico y manejo de los síntomas respiratorios recurrentes en niños (Appachi et al., 2017; Galligan et al., 2018).

Guatemala es un país en vías de desarrollo, donde las prioridades en salud pediátrica están concentradas en erradicar la desnutrición y en implementar programas de vacunación efectivos. Por tal motivo, no se había hecho esfuerzo alguno para desarrollar una unidad aerodigestiva, ya que se planteaba como un tratamiento distante a nuestra realidad.

No fue hasta el año 2018 cuando se desarrolló la primera unidad aerodigestiva pediátrica (UAP) en un hospital privado de la Ciudad de Guatemala. Al ser la única de este tipo en el país, no se contaba con datos locales al respecto; por lo que el aporte científico de esta investigación permitió describir las características y el manejo multidisciplinario de pacientes pediátricos con problemas aerodigestivos de alta complejidad; atendidos en la UAP de enero del año 2018 a mayo del 2019.

\section{Pacientes y métodos}

Diseño. Se llevó a cabo un estudio retrospectivo cuantitativo, cuyo objetivo general fue determinar las características de los pacientes evaluados en la UAP. Se tomó en cuenta variables descriptivas: edad, sexo, indicación de procedimiento, procedimiento realizado (laringoscopia, broncoscopia, endoscopia digestiva alta, $\mathrm{pH}$ metría con impedancia esofágica), y estudios diagnósticos complementarios como toma de biopsia de vía aérea superior e inferior, cultivo de lavado broncoalveolar, toma de biopsia esófago gástrica, y diagnósticos finales.

Población y criterios de inclusión de estudio. Se contó con una población de 69 pacientes en edad pediátrica ( 0 a 21 años de edad) con sintomatología persistente de la vía aérea, representando el total de casos reportados en la UAP desde el 1 de enero de 2018 hasta el 31 de mayo de 2019.

Instrumentos. Fueron utilizados 69 informes finales con los datos personales de cada paciente, procedimiento(s) realizado(s) y diagnósticos, obtenidos de la base de datos de las diferentes áreas de la UAP: unidad de endoscopias, laboratorio clínico y microbiológico, y laboratorio de patología.

Análisis de datos. En una base de datos se registraron los datos de cada paciente incluyendo: edad, sexo, indicación del procedimiento, procedimiento realizado, hallazgos y diagnóstico sustentados por: laringoscopia, broncoscopia, endoscopia digestiva alta, impedancia esofágica con pH metría, toma de biopsias, lavado broncoalveolar, y estudios de microbiología y serología, según como se describen en los registros médicos de cada paciente. Se realizó la tabulación de los datos en cuadros de Microsoft Excel 2010 para el desarrollo del análisis estadístico en el programa SPSS versión 24, con licencia autorizada. Finalmente, se presenta el resultado del análisis realizado con la finalidad de demostrar el valor diagnóstico de la intervención multidisciplinaria de cada caso.

\section{Resultados}

Se reportaron resultados de los 69 pacientes evaluados en la UAP; cuya edad variaba desde días de vida, hasta pacientes de 21 años. De estos pacientes, el 62 \% son de 5 años o menores (Tabla 1).

Las principales indicaciones para que los pacientes fueran evaluados en la UAP fueron: tos húmeda recurrente (20\%), crup recurrente (18\%), y bronquitis bacteriana recurrente (14\%) (Tabla 2).

Fueron realizadas 69 laringoscopias en las cuales el diagnóstico más frecuente fue laringomalacia en distintos grados (25\%) (Tabla 3). Asimismo, se realizaron 69 broncoscopias en las cuales el diagnóstico más frecuente fue bronquitis bacteriana recurrente con $52 \%$, seguida de traqueomalacias en distintos grados (22\%) (Tabla 3). Algunos pacientes tuvieron dos o más diagnósticos, por lo cual la cifra final de diagnósticos realizados mediante broncoscopia es 86 . Los patógenos más frecuentes evidenciados por cultivo de lavado broncoalveolar fueron Haemophilus influenzae (20\%), Moraxella catarrhalis (10\%) y Streptococcus pneumoniae (8\%) (Tabla 4) estando presentes aislados o en coinfección en algunos pacientes. 
Se efectuaron 45 endoscopias digestivas altas que corresponden al $65 \%$ de los pacientes evaluados. Algunos pacientes tuvieron dos o más diagnósticos, por lo cual la cifra final de diagnósticos realizados mediante endoscopias digestivas altas es 80 . A 44 de los 45 pacientes se les tomó muestras para biopsias esofágicas, gástricas y duodenales. En las cuales el diagnóstico más frecuente fue enfermedad por reflujo gastroesofágico (41 \%) (Tabla 3), en 12 casos se confirmó el diagnóstico mediante la asociación de $\mathrm{pH}$ metría con impedancia esofágica (Tabla 4) y en 30 casos mediante biopsia esofágica (Tabla 4). Es importante mencionar que los pacientes con diagnóstico de enfermedad por reflujo gastroesofágico presentaban síntomas clínicos sugestivos de la patología, los hallazgos en la endoscopia fueron consistentes con cambios crónicos y agudos de esofagitis y gastritis erosiva, se realizó una correlación clínico - patológica con los resultados de $\mathrm{pH}$ metría y/o hallazgos histológicos de las biopsias.

Finalmente, se evidenció que el $79 \%$ de los pacientes presentó más de una patología de diferente etiología con diagnóstico por diferentes especialidades (Tabla 5), siendo los diagnósticos de bronquitis bacteriana recurrente y enfermedad por reflujo gastroesofágico los que con mayor frecuencia fueron reportados como comorbilidades en un mismo paciente (Tabla 3).
Tabla 1: Edades agrupadas de pacientes de unidad aerodigestiva pediátrica.

\begin{tabular}{|c|c|c|}
\hline Edad en años & $\begin{array}{c}\text { Frecuencia de } \\
\text { Pacientes }\end{array}$ & $\begin{array}{c}\text { Porcentaje } \\
\%\end{array}$ \\
\hline 0 a 2 & 19 & 27 \\
\hline 3 a 5 & 24 & 35 \\
\hline 6 a 11 & 15 & 22 \\
\hline Más de 11 & 11 & 16 \\
\hline Total & 69 & 100 \\
\hline
\end{tabular}

Tabla 2: Indicaciones para evaluación de pacientes en unidad aerodigestiva pediátrica.

\begin{tabular}{lcc}
\hline Indicación & $\begin{array}{c}\text { Frecuencia de } \\
\text { Pacientes }\end{array}$ & $\begin{array}{c}\text { Porcentaje } \\
\%\end{array}$ \\
\hline Tos húmeda recurrente & 14 & 20 \\
Crup recurrente & 13 & 18 \\
Bronquitis bacteriana recurrente & 10 & 14 \\
Bronquitis crónica & 6 & 8 \\
Tos seca crónica & 6 & 8 \\
Neumonía recurrente & 5 & 7 \\
Bronconeumonía recurrente & 4 & 5 \\
Espasmo laríngeo & 3 & 4 \\
Apnea & 2 & 3 \\
Otros & 10 & 13 \\
\hline Total & $\mathbf{7 3}$ & $\mathbf{1 0 0}$ \\
\hline
\end{tabular}

Tabla 3: Total de diagnósticos de pacientes de unidad aerodigestiva pediátrica.

\begin{tabular}{|c|c|c|c|c|c|c|c|c|}
\hline Laringoscopia & $\begin{array}{c}N^{\circ} \text { de } \\
\text { Pacientes }\end{array}$ & $\begin{array}{c}\text { Porcentaje } \\
\%\end{array}$ & Broncoscopia & $\begin{array}{c}N^{\circ} \text { de } \\
\text { Pacientes }\end{array}$ & Porcentaje & $\begin{array}{c}\text { Endoscopia di- } \\
\text { gestiva alta + pH } \\
\text { metría + Biopsia } \\
+ \text { Clínica } \\
\end{array}$ & $\begin{array}{c}N^{\circ} \text { de } \\
\text { Pacientes }\end{array}$ & $\begin{array}{c}\text { Porcentaje } \\
\quad \%\end{array}$ \\
\hline Normal- No hallazgos & 44 & 63 & $\begin{array}{l}\text { Bronquitis bacteriana } \\
\text { recurrente }\end{array}$ & 44 & 52 & $\begin{array}{c}\text { Enfermedad por } \\
\text { Reflujo Gastroeso- } \\
\text { fágico }\end{array}$ & 33 & 41 \\
\hline Laringotraqueomalacia & 6 & 9 & $\begin{array}{l}\text { Traqueomalacia } \\
\text { grado I }\end{array}$ & 13 & 16 & Gastritis crónica & 18 & 22 \\
\hline Laringomalacia grado I & 5 & 8 & Broncomalacia & 10 & 11 & $\begin{array}{c}\text { Duodenitis crónica } \\
\text { leve }\end{array}$ & 16 & 20 \\
\hline Laringomalacia grado II & 5 & 8 & $\begin{array}{l}\text { Traqueomalacia } \\
\text { grado II }\end{array}$ & 5 & 6 & $\begin{array}{l}\text { Gastritis crónica } \\
\text { por H.pylori }\end{array}$ & 7 & 9 \\
\hline Granuloma laríngeo & 2 & 3 & Bronquitis crónica & 4 & 5 & $\begin{array}{c}\text { Gastritis superficial } \\
\text { erosiva }\end{array}$ & 4 & 5 \\
\hline $\begin{array}{l}\text { Hiperplasia linfoide benig- } \\
\text { na de la base de la lengua }\end{array}$ & 2 & 3 & Traqueítis & 3 & 3 & Esófago de Barrett & 2 & 3 \\
\hline Laringomalacia grado III & 2 & 3 & Bronquiectasia & 1 & 1 & & & \\
\hline Laringitis & 1 & 1 & Bronquio accesorio & 1 & 1 & & & \\
\hline Papilomatosis laríngea & 1 & 1 & Bronquio traqueal & 1 & 1 & & & \\
\hline \multirow[t]{4}{*}{ Quiste de cuerda vocal } & 1 & 1 & Divertículo traqueal & 1 & 1 & & & \\
\hline & & & Hemorragia bronquial & 1 & 1 & & & \\
\hline & & & $\begin{array}{c}\text { Neumonía atelectásica } \\
\text { purulenta }\end{array}$ & 1 & 1 & & & \\
\hline & & & $\begin{array}{c}\text { Neumonía apical } \\
\text { derecha }\end{array}$ & 1 & 1 & & & \\
\hline Total & 69 & $100 \%$ & & 86 & $100 \%$ & & 80 & $100 \%$ \\
\hline
\end{tabular}


Tabla 4: Estudios complementarios de pacientes de la unidad aerodigestiva pediátrica.

\begin{tabular}{|c|c|c|}
\hline & Frecuencia de Pacientes & Porcentaje \% \\
\hline \multicolumn{3}{|l|}{ Resultados cultivos bacteriológicos de Lavados broncoalveolares } \\
\hline H. influenzae & 13 & 20 \\
\hline S. mitis & 11 & 17 \\
\hline M. catarrhalis & 6 & 10 \\
\hline S. pneumoniae & 5 & 8 \\
\hline P. aeruginosa & 3 & 5 \\
\hline S. parasanguinis & 3 & 5 \\
\hline S. pyogenes & 3 & 5 \\
\hline S. aureus & 2 & 4 \\
\hline Otros & 9 & 15 \\
\hline Negativo & 7 & 11 \\
\hline Total & 62 & $100 \%$ \\
\hline \multicolumn{3}{|l|}{ Ph metrías e Impedancias Esofágicas } \\
\hline Resultados positivos para reflujo gastroesofágico asociado a tos & 12 & 60 \\
\hline Resultados negativos para reflujo gastroesofágico asociado a tos & 8 & 40 \\
\hline Total & 20 & $100 \%$ \\
\hline \multicolumn{3}{|l|}{ Resultados biopsias esofágicas } \\
\hline Esofagitis péptica & 30 & 75 \\
\hline Sin alteraciones histológicas & 8 & 20 \\
\hline Esófago de Barrett & 2 & 5 \\
\hline Total & 40 & $100 \%$ \\
\hline
\end{tabular}

Tabla 5: Pacientes de unidad aerodigestiva pediátrica con patología simple o múltiple.

\begin{tabular}{|c|c|c|c|}
\hline & Patología & Frecuencia de Pacientes & Porcentaje \\
\hline & Pulmonar & 14 & 20 \\
\hline \multirow[t]{3}{*}{ Una única especialidad } & Otorrinolaringológica & 1 & 1 \\
\hline & Gastroenterológica & 0 & 0 \\
\hline & Total & 15 & $21 \%$ \\
\hline Dos especialidades & Otorrinolaringológica + pulmonar & 20 & 30 \\
\hline Tres especialidades & $\begin{array}{l}\text { Otorrinolaringológica + pulmonar + } \\
\text { gastroenterológica }\end{array}$ & 34 & 49 \\
\hline & Total & 69 & $100 \%$ \\
\hline
\end{tabular}

\section{Discusión}

De los pacientes evaluados en la UAP existe un predominio de edad de 5 años o menor, lo cual va acorde con lo reportado por otros programas aerodigestivos como el del Centro Infantil de la Clínica Mayo en Estados Unidos (Boesch et al., 2018). La evaluación de este grupo etario es posible gracias a que la broncoscopia, que constituye uno de los avances más significativos en el manejo de las enfermedades respiratorias, ha ido evolucionando y actualmente permite realizar procedimientos en pacientes a corta edad. Esto permite un diagnóstico temprano y evidenciar patologías que a largo plazo producirán problemas en el crecimiento, desarrollo y evolución clínica del paciente, favoreciendo así un enfoque diagnóstico integral.

Los pacientes fueron previamente evaluados por al menos un especialista (pediatra, neumólogo, otorrinolaringólogo, gastroenterólogo) y recibieron pruebas terapéuticas con antibioterapia sin mejoría clara en su cuadro clínico. La tos es un síntoma común de patología respiratoria, y es importante considerar la triple endoscopia en aquellos pacientes que no mejoran con tratamiento con antihistamínicos, antitusivos y/o antibióticos orales de primera línea 
como amoxicilina en las subsecuentes evaluaciones clínicas; o más aún en aquellos que ya han recibido varios ciclos de antibioterapia. Cabe recalcar que en los pacientes evaluados por una UAP la etiología de la tos se considera multifactorial, y que puede pasarse por alto comorbilidades si únicamente se realiza un procedimiento.

El hallazgo laringoscópico predominante de laringomalacia no debe pasarse por alto, ya que; al ser una patología estructural congénita puede ser una importante causa o comorbilidad de problemas de deglución, reflujo gastroesofágico, fallo para progresar y tos crónica (Naime et al., 2018). Esta patología es la más frecuente en las anomalías congénitas de la laringe y se considera que resolverá de forma espontánea en casi todos los casos. Sin embargo, su incidencia es alta en los pacientes con síntomas respiratorios crónicos, por lo que es útil conocer su existencia, ya que; condiciona la gravedad, duración y enfoque terapéutico de afecciones comunes de la infancia como es el caso de infecciones virales de vía aérea superior.

Las broncoscopias brindaron información importante respecto a la etiología de la sintomatología del paciente, como es el caso del hallazgo de bronquitis bacteriana recurrente. Tomando en cuenta que la bronquitis bacteriana prolongada es la primera causa de tos húmeda persistente en niños menores de 5 años, el diagnóstico microbiológico es fundamental para una mejor orientación terapéutica en función de la persistencia del proceso bronquial (Naime et al., 2018). Los patógenos más frecuentes evidenciados mediante cultivo de lavado broncoalveolar fueron muy similares a los reportados como principales entidades en otros estudios, por ejemplo, en el informe del Sistema Nacional de Salud de Michigan (Fracchia et al., 2018). De los pacientes evaluados en la UAP se reportan casos de Haemophilus influenzae no tipificable con pacientes con adecuado estado de inmunización respecto a Haemophilus influenza tipo B. Es importante mencionar que Moraxella catarrhalis se considera un patógeno colonizador de la vía aérea pediátrica y de etiología frecuente en otitis media aguda y rinosinusitis; sin embargo, se tiene por infrecuente como patógeno de neumonía adquirida en la comunidad. De acuerdo con los datos recabados en este estudio se sugiere que este patógeno tiene un rol más activo en enfermedad bronquial, datos similares a informes de otras UAP (Aebi, 2011).

De acuerdo con los programas aerodigestivos pediátricos sugeridos en consenso por múltiples centros médicos en Estados Unidos se debe realizar la triple endoscopia en todos los pacientes (Boesch et al., 2018). Aun así, debido a limitaciones económicas por parte de los pacientes y considerando que el predominio de los síntomas era respiratorio, en $35 \%$ de casos del estudio se priorizó realizar únicamente los procedimientos de la vía aérea. Sin embargo, los hallazgos de las endoscopias digestivas altas asociados a las $\mathrm{pH}$ metrías y/o biopsias evidenciaron la importante presencia de reflujo gastroesofágico en estos pacientes. Según la perspectiva de otorrinolaringología pediátrica, la tos crónica se atribuye frecuentemente a reflujo gastroesofágico; a pesar de ello, consideran que el edema y eritema que se observa en las estructuras de la laringe son signos no específicos y que generalmente se atribuyen de forma errónea a dicha patología. En su contraparte, en la perspectiva de gastroenterología pediátrica el reflujo gastroesofágico es el desorden gastrointestinal que con mayor frecuencia se presenta como tos crónica, por lo que se debe considerar la historia clínica y evolución de los pacientes para conducir la terapia farmacológica; decisión que se puede valorar de mejor manera al estar presente el equipo multidisciplinario al momento de realizar la triple endoscopia y la evaluación como unidad aerodigestiva pediátrica (Naime et al., 2018).

\section{Conclusión}

Este estudio concluye la utilidad de la triple endoscopia tanto en el diagnóstico como en el manejo de las patologías aerodigestivas pediátricas de alta complejidad; así mismo su utilidad al evidenciar que múltiples etiologías pueden contribuir a síntomas crónicos de la vía aérea. Al ser este el primer estudio al respecto en Guatemala, se considera la piedra angular para futuras investigaciones y motivar a la replicación del modelo de unidad aerodigestiva pediátrica en otros centros de atención, $y$, de esta manera, contribuir a mejorar la salud y proporcionar atención costo/eficiente con menor riesgo de exposición anestésica y radioactiva a la población pediátrica latinoamericana.

\section{Ética de la investigación}

En el presente estudio se tuvo acceso a los datos generales de cada paciente, incluyendo el nombre completo. Al no tener un consentimiento informado mediante el cual los pacientes autoricen ser identificados por su nombre, éste no se revela ni se utilizó en la base de datos. Así mismo, por tratarse de un estudio descriptivo, no se sometió a los pacientes a ningún otro procedimiento, como toma de muestras sanguíneas o administración de medicamentos, excepto por lo descrito en el expediente clínico. Tampoco se manipularon las biopsias ni estudios complementarios realizados en la unidad aerodigestiva pediátrica. El presente estudio contó con la aprobación del comité de ética de la Universidad Francisco Marroquín.

\section{Fuentes de financiamiento}

Los resultados de esta investigación no se utilizaron ni se utilizarán con fines de lucro y no se recibió patrocinio alguno para su realización. 


\section{Contribuciones y conflictos declarados por los autores}

La recolección de datos, la redacción del manuscrito y la evaluación estadística fueron realizadas por la Dra. Rosina López. El diseño del estudio, análisis de resultados y la revisión de la redacción del manuscrito fueron realizados por los doctores Maynor Bravo y Shirley Cuan. La realización de esta investigación fue asesorada por el Dr. Edgar Beltetón, quien a su vez contribuyó en el análisis de los resultados. El Dr. Christian Farrington contribuyó en el análisis de los diagnósticos de gastroenterología. Los autores declaran no tener conflictos de intereses.

\section{Referencias}

Aebi C. (2010) Moraxella catarrhalis - Pathogen or Commensal? In: Curtis N, Finn A, Pollard A. Hot Topics in Infection and Immunity in Children VII. Advances in Experimental Medicine and Biology 697, 107-116.

Appachi S, Banas A, Feinberg L, Henry D, Kenny D, Kraynack N, Carl J \& Krakovitz P. (2017.) Association of Enrollment in an Aerodigestive Clinic With Reduced Hospital Stay for Children With Special Health Care Needs. JAMA Otolaryngology-Head \& Neck Surgery 143, 1117-1121.

Boesch RP, Balakrishnan K, Grothe RM, Driscoll SW, Knoebel EE, VIsscher SL \& Cofer SA. (2018). Interdisciplinary aerodigestive care model improves risk, cost, and efficiency. International Journal of Pediatric Otorhinolaryngology 113, 119-123.

Boesch RP, Balakrishnan K, Acra S, Benscoter DT, Cofer SA, Collaco JM, Dahl JP, Daines CL, DeAlarcon A, DeBoer EM, Deterding RR, Friedlander JA, Gold BD, Grothe RM, Hart CK, Kazachkov M, Lefton-Greif MA, Miiller CK, Moore PE, Pentiuk S, Peterson-Carmichael S, Piccione K, Prager JD, Putnam PE, Rosen R, Rutter MJ, Ryan MJ, Skinner ML, Torres-Silva C, Wooten CT, Zuk KB, Cotton RT \& Wood RE. (2018). Structure and Functions of Pediatric Aerodigestive Programs: A Consensus Statement. Pediatrics 141, e20171701.
Collaco JM, Aherrera AD, Au Yeung KJ, Lefton-Greif MA, Hoch J \& Skinner ML. (2017). Interdisciplinary Pediatric Aerodigestive Care and Reduction in Health Care Costs and Burden. JAMA Otolaryngology-Head \& Neck Surgery 141, 101-105.

Fracchia MS, Diercks G, Cook A, Hersh C, Hardy S, Hartnick M \& Hartnick C. (2019) The diagnostic role of triple endoscopy in pediatric patients with chronic cough. International Journal of Pediatric Otorhinolaryngology 116, 58-61.

Galligan MM, Bamat TW, Hogan AK \& Piccione J. (2018). The Pediatric Aerodigestive Center as a Tertiary Care-Based Medical Home: A Proposed Model. Current Problems in Pediatric and Adolescent Health Care 48, 104-110.

Kumar S, Choi S \& Gupta SK. (2019). Eosinophilic Esophagitis-A Primer for Otolaryngologists. JAMA Otolaryngology-Head \& Neck Surgery $145,373-380$

Naime S, Batra SK, Fiorillo C, Collins ME, Gatti M, Krakovsky GM, Sehgal S, Bauman NM \& Pillai DK. (2018). Aerodigestive Approach to Chronic Cough in Children. Current Treatment Options in Pediatrics 4, 467-479.

Rotsides JM, Krakovsky GM, Pillai DK, Sehgal S, Collins ME, Noelke CE \& Bauman NM. (2017). Is a Multidisciplinary Aerodigestive Clinic More Effective at Treating Recalcitrant Aerodigestive Complaints Than a Single Specialist? Annals of Otology, Rhinology \& Laryngologyog 126, 537-543.

Skinner ML, Lee SK, Collaco JM, Lefton-Greif MA, Hoch J \& Au Yeung KJ. (2016). Financial and Health Impacts of Multidisciplinary Aerodigestive Care. Otolaryngoly- and Neck Surgery 154, 1064-1067. 